\title{
Effect of cytostatic proline rich polypeptide-1 on tumor suppressors of inflammation pathway signaling in chondrosarcoma
}

\author{
KARINA GALOIAN $^{1}$, SHIHUA LUO ${ }^{1}$, AMIR QURESHI ${ }^{1}$, PARTHIK PATEL ${ }^{1}$, RACHEL PRICE ${ }^{1}$, \\ ASHLYN S. MORSE ${ }^{1}$, GOR CHAILYAN ${ }^{2}$, SILVA ABRAHAMYAN $^{2}$ and H. T. TEMPLE ${ }^{3}$ \\ ${ }^{1}$ Department of Orthopaedics, University of Miami, Miller School of Medicine, Miami, FL 33136, USA; \\ ${ }^{2}$ Buniatian Institute of Biochemistry Academy of Sciences of Armenia, Yerevan 0014, Armenia; \\ ${ }^{3}$ Center for Translational Research, Nova Southeastern University, Fort Lauderdale, FL 33314, USA
}

Received June 14, 2016; Accepted August 11, 2016

DOI: $10.3892 / \mathrm{mco} .2016 .1010$

\begin{abstract}
Cytokines produced in thetumourmicroenvironment exert an important role in cancer pathogenesis and in the inhibition of disease progression. Cancer of the cartilage is termed metastatic chondrosarcoma; however, the signaling events resulting in mesenchymal cell transformation to sarcoma have yet to be fully elucidated. The present study aimed to characterize the cytokine expression profile in the human JJ012 chondrosarcoma cell line, as well as the effect of cytostatic proline-rich polypeptide-1 (PRP-1). Western blot experiments demonstrated that the levels of suppressor of cytokine signaling 3 (SOCS3) were upregulated in chondrocytes compared with chondrosarcoma cells. Addition of PRP-1 restored the expression of the tumor suppressors, SOCS3 and ten-eleven-translocation methylcytosine dioxygenase 1 and 2 (TET1/2), in a dose-responsive manner. It is known that methylation of histone $\mathrm{H} 3 \mathrm{~K} 9$ was eliminated from the promoters of the inflammation-associated genes. PRP-1 inhibited H3K9 demethylase activity with an $\mathrm{IC}_{50}$ (concentration required to give half-maximal inhibition) value of $3.72 \mu \mathrm{g} / \mathrm{ml}$ in the chondrosarcoma cell line. Data obtained from ELISA experiments indicated that the expression of interleukin-6 (IL-6) in chondrosarcoma cells was 86-fold lower compared with that in C28 chondrocytes. In the present study, a 53-fold downregulation of IL-6 expression in co-culture of chondrosarcoma cells and C28 chondrocytes was identified as well. Downregulation of IL-6 expression has been documented in numerous other tumor types, although the reasons for this have not been fully
\end{abstract}

Correspondence to: Dr Karina Galoian, Department of Orthopaedics, University of Miami, Miller School of Medicine, 1600 NW 10th Avenue, Miami, FL 33136, USA

E-mail: kgaloian@med.miami.edu

Key words: chondrosarcoma, proline-rich polypeptide-1, interleukin-6, inflammation, suppressor of cytokine signaling 3 established. In chondrosarcoma, IL-6 manifests itself as an anti-inflammatory agent and, possibly, as an anti-tumorigenic factor. To explore protein-DNA interactions leading to such differences, a gel-shift chemiluminescent assay was performed. Gel shifts were observed for chondrosarcoma and chondrocytes in the lanes that contained nuclear cell extract and oligo-IL-6 DNA. Notably, the DNA-protein complexes in C28 chondrocytes were markedly larger compared with those in chondrosarcoma cells. The mechanisms that underpin such differences, and characterization of the interacting proteins, remain to be fully elucidated.

\section{Introduction}

It is now widely accepted that the risk of cancer may increase as a result of chronic inflammation, and that cytokines and growth factors have very important roles in this process. Cytokines are secreted proteins necessary for the co-ordination of immune responses, homeostasis, cell communications and cancer, which is a cell type context-dependent process.

Understanding the involvement of certain cytokines in the failed differentiation programs that lead to cancer or a loss of tumor-suppressive functions is undoubtedly one of the challenges in modern molecular immuno-oncology (1). The inflammatory cells and regulators may facilitate angiogenesis and promote the growth, invasion and metastasis of tumor cells. Previous research regarding inflammation-associated cancer development has focused on cytokines and chemokines, as well as their downstream targets in linking inflammation and cancer.

Chondrosarcoma is cancer of the cartilage. It is the second most common primary bone malignancy, which affects the pelvis, long bones and spine, as well as the larynx and the head and neck, which eventually metastasizes. The signaling events resulting in mesenchymal cell transformation to sarcoma have yet to be fully elucidated. Chondrosarcoma does not respond to chemotherapy or radiation; therefore, the search for novel therapies is very urgent (2).

This study aimed to characterize the cytokine expression profile in the human JJ012 chondrosarcoma cell line compared 
with C28 chondrocytes. The effect of the mammalian target of rapamycin complex 1 (mTORC1) inhibitor and anti-proliferative immunomodulator, proline-rich polypeptide 1 (PRP-1) (3-7), on the expression of cytokines, tumor suppressors SOCS3, TET1/2 and oncoproteins of inflammatory oncogenic pathways, including the Hedgehog and Hippo pathways, was also investigated. PRP-1 is a powerful upregulator of tumor suppressor microRNAs (miRNAs) and proteins, and a downregulator of oncoproteins, as reported in previous publications from our laboratory (8-10). Ten eleven translocation (TET) enzymes, a family of $\alpha$-ketoglutarate $(\alpha-K G)$-dependent dioxygenases, catalyse the oxidative reactions of 5-methylcytosine $(5 \mathrm{mC})$ to promote the DNA demethylation process. TET (methylcytosine dioxygenase) enzyme activity is inhibited in IDH1/2-mutated tumors by the oncometabolite, 2-hydroxyglutarate, an antagonist of $\alpha-\mathrm{KG}$ linking $5 \mathrm{mC}$ oxidation with cancer development (11-16). Increased levels of D2 hydroxyglutarate (D-2-HG) have been identified in cartilage tumors that possess an IDHI or an IDH2 mutation (17). A recent report has demonstrated that TET-1 and -2 are present in human chondrocytes, and that TET1 expression was markedly reduced by inflammatory factors (18). Hippo and Hedgehog signaling contribute to malignancies of mesenchymal origin. These two pathways are associated with inflammation, and promote tumorigenesis in numerous diseases, including soft tissue sarcomas, chondrosarcomas, and so forth (19-23). The Hedgehog pathway is associated with inflammation and cancerogenesis (24-26). The network of Hippo signaling regulates the specific enrichment of genes involved in immune and inflammatory responses (27).

\section{Materials and methods}

Tissue culture. The complete growth medium for the human JJ012 chondrosarcoma cells (obtained from Dr Joel Block's Laboratory, Rush University, Chicago, IL, USA) and C28 chondrocytes comprised the following: Modified Eagle's medium (DMEM)/MEM (Thermo Fisher Scientific, Inc., Waltham, MA, USA) supplemented with F12, 10\% fetal bovine serum (FBS; Thermo Fisher Scientific, Inc.), $25 \mu \mathrm{g} / \mathrm{ml}$ ascorbic acid, $100 \mathrm{ng} / \mathrm{ml}$ insulin, $100 \mathrm{nM}$ hydrocortisone and $1 \%$ penicillin/streptomycin.

Growth medium for the bone marrow-derived mesenchymal stem cells (ATCC ${ }^{\circledR}$ PCS-500-012; American Type Culture Collection, Manassas, VA, USA), Mesenchymal Stem Cell Basal medium, comprised the following: Fetal bovine serum, $485 \mathrm{ml}, 7 \%$ (v/v); recombinant human (rh) insulin-like growth factor-1, $15 \mathrm{ng} / \mathrm{ml}$; rh basic fibroblast growth factor, $125 \mathrm{pg} / \mathrm{ml}$; L-alanyl-L-glutamine, $2.4 \mathrm{mM}$; gentamicin, $10 \mu \mathrm{g} / \mathrm{ml}$; amphotericin $\mathrm{B}, 0.25 \mu \mathrm{g} / \mathrm{ml}$; and penicillin/streptomycin, $1 \%(\mathrm{v} / \mathrm{v})$.

Enzyme-linked immunosorbent assay (ELISA). A Human Inflammatory Cytokines Multi-Analyte ELISArray kit (cat. no. MEH-004A; Qiagen, Valencia, CA, USA) was used to detect a panel of 12 cytokines following a conventional ELISA protocol, according to the manufacturer's protocol. The ELISA panel included: Interleukin (IL)- $1 \alpha$, IL-1 $\beta$, IL-2, IL-4, IL-6, IL-8, IL-10, IL-12, IL-17A, interferon- $\gamma$ (IFN- $\gamma$ ), tumor necrosis factor- $\alpha$ (TNF- $\alpha)$ and granulocyte-macrophage colony-stimulating factor (GM-CSF).

Polyacrylamide gel electrophoresis and western blotting. Upon confluency, the cells were trypsinized and seeded in 6 -well cluster dishes at a concentration of $1 \times 10^{6}$ cells $/ \mathrm{ml}$. The experimental samples were treated with PRP-1 in corresponding concentrations, whereas control samples were not treated with the peptide. The cells were incubated for $24 \mathrm{~h}$ in a $5 \% \mathrm{CO}_{2}$ incubator at $37^{\circ} \mathrm{C}$. On the following day, the cells were washed with ice-cold phosphate-buffered saline. A protease inhibitor (P8340; Sigma-Aldrich, St. Louis, MO, USA) was added to the cell lysis buffer (C2978; Sigma-Aldrich) in a 1:100 ratio. The cells were collected with a scraper and centrifuged at $15,000 \mathrm{x} g$ at $4^{\circ} \mathrm{C}$. The samples were loaded and run according to the manufacturer's protocol. The gel running time was $\sim 1 \mathrm{~h}$ at $100 \mathrm{~V}$, after which the gel was transferred for western blotting. PVDF membranes were briefly treated in methanol, distilled water and transfer buffer prior to the western blot transfer, which was performed in a cold room for a further hour.

The membranes were then subjected to incubation with blocking buffer on the rocker at room temperature for $1 \mathrm{~h}$. Subsequently, the membranes were incubated with primary antibodies overnight in the cold room. All primary and secondary antibodies were diluted in the identical blocking buffer. The following day, the membranes were washed three times (15 min each wash) in Tween/PBS wash buffer and subjected to incubation with secondary antibodies for $2 \mathrm{~h}$ at room temperature, followed by three consecutive washes. Enhanced chemiluminescence (ECL) reagent was applied to the membrane according the manufacturer's protocol, and $\mathrm{X}$-ray film was developed in the dark room following exposure for $2-10 \mathrm{~min}$, depending on the experiment.

Reagents were purchased from Lonza, Inc. (Walkersville, MD, USA), and all the associated procedures were performed according to the manufacturer's protocol. The catalog numbers for the reagents and the suppliers are listed as follows: Pager gold precast gels (cat. no. 59502; 4\% stack, 10\% TRIS-glycine; Lonza, Inc.); ECL reagent (cat. no. RPN2109; GE Healthcare, Little Chalfont, UK); Western blocker solution (cat. no. W0138; Sigma-Aldrich); ProSieve ${ }^{\mathrm{TM}}$ QuadColor $^{\mathrm{TM}}$ Protein Markers (4.6-300 kDa, cat. no. 00193837; Lonza, Inc.); 20X reducing agent for ProSieve ${ }^{\mathrm{TM}}$ ProTrack $^{\mathrm{TM}}$ dual color loading buffer (cat. no. 00193861; Lonza, Inc.); EX running buffer (cat. no. 00200307; Lonza, Inc.); ProSieve ${ }^{\mathrm{TM}}$ EX Western Blot Transfer buffer (cat. no. 00200309; Lonza, Inc.); and Immobilon ${ }^{\circledR}$-P Polyvinylidene difluoride membranes (cat. no. P4188; Sigma-Aldrich). The primary antibodies used were as follows: Rabbit polyclonal anti-suppressor of cytokine signaling 3 (socs3) antibody [cat. no. ab16030, molecular weight (M.W.) $30 \mathrm{kDa}$; Abcam, Cambridge, UK]; rabbit polyclonal anti-transforming growth factor- $\beta$ (TGF- $\beta$ )-1/-2/-3 (H-112; cat. no. sc-7892, M.W. 12-25 kDa); anti-Smad 2 rabbit polyclonal antibody (cat. no. SAB4300562, M.W. 52 kDa; SABiosciences, Frederick, MD, USA); anti-Stat3 mouse monoclonal antibody (mAb; cat. no. sc-293151, M.W. 86-91 kDa, Santa Cruz Biotechnology, Inc., Santa Cruz, CA, USA); mouse monoclonal anti-tubulin (M.W. $52 \mathrm{kDa}$; cat. no. T5168; Sigma-Aldrich); The secondary antibodies were as 
follows: Anti-mouse immunoglobulin G (IgG; cat. no. A9044; Sigma-Aldrich); goat anti-rabbit IgG peroxidase conjugate (cat. no. A0545; Sigma-Aldrich); anti-TET1 rabbit polyclonal antibody (cat. no. ab105475, M.W. 235 kDa; Abcam); and anti-TET2 rabbit polyclonal antibody (cat. no. ab94580, M.W. $224 \mathrm{kDa}$, Abcam). All primary antibodies were used at a dilution of 1:1,000; the secondary antibodies were used at a dilution of 1:5,000.

Hippo and Hedgehog signaling antibodies. A Hippo Signaling Antibody Sampler kit (cat. no. \#8579; Cell Signaling Technology, Inc., Danvers, MA, USA) was used for experiments performed in the present study. The kit comprised the following: Anti-p-Yap (Ser387) (D1E7Y) rabbit mAb (cat. no. \#13619, M.W 75 kDa); anti-LATS1 (C66B5) rabbit mAb (cat. no. \#3477, M.W. $140 \mathrm{kDa}$ ); anti-p-MOB1 (Thr35) (D2F10) rabbit mAb (cat. no. \#8699, M.W. 24 kDa); anti-MOB1 (E1N9D) rabbit mAb (cat. no. \#13730, M.W. $25 \mathrm{kDa}$ ); anti-macrophage stimulating 1 (Mst1) rabbit mAb (cat. no. \#3682, M.W. 59 kDa); anti-Mst2 rabbit mAb (cat. no. \#3952, M.W. 60 kDa); anti-SAV1 (D6M6X) rabbit mAb (cat. no. \#13301, M.W. 45 kDa); anti-p-Yap (Ser127) (D9W21) rabbit mAb (cat. no. \#13008, M.W. 65-75 kDa); anti-Yap/Taz (D24E4) rabbit mAb (cat. no. \#8418, M.W. 50-70 kDa); and the secondary antibody, horseradish peroxidase (HRP) -linked goat anti-rabbit IgG (cat. no. \#7074).

A Hedgehog Signaling Antibody Sampler kit (cat. no. \#8358; Cell Signaling Technology, Inc.) was also used, comprising the following antibodies: Anti-Sonic Hedgehog (Shh) (C9C5) rabbit mAb (cat. no. \#2207, M.W. 19-45 kDa); anti-Patch homology 1 (PTCH1) (C53A3) rabbit mAb (cat. no. \#2468, M.W. 180-210 kDa); anti-PTCH2 (G1191) rabbit mAb (cat. no. \#2479, M.W $130 \mathrm{kDa}$ ); anti-suppressor of fused homolog (SUFU) (C54G2) rabbit mAb (cat. no. \#2520, M.W. 54 kDa); anti-Gli1 (C68H3) rabbit mAb (cat. no. \# 3538, M.W $160 \mathrm{kDa}$ ); and the secondary antibody, HRP-linked goat anti-rabbit IgG (cat. no. \#7074).

Nuclear extraction. A nuclear extraction kit (cat. no. \#40010; Active Motif, Carlsbad, CA, USA) was used to prepare the nuclear extracts from the chondrosarcoma, adult mesenchymal stem and chondrocytic C28 cells, according to the manufacturer's protocol.

Gel-shift/gel retardation assay. A Gelshift ${ }^{\mathrm{TM}}$ Chemiluminescent electrophoretic mobility shift assay (EMSA) kit (cat. no. \#37341; Active Motif) was used to analyze the protein-DNA interactions. The principle behind EMSA relies on the fact that DNA-protein complexes migrate slower than DNA alone in a native polyacrylamide or agarose gel. The difference in electrophoretic separation of DNA-protein complexes can be visualized as a 'shift' in migration of the labeled DNA band. Briefly, nuclear extracts were incubated with a biotin 3'- or 5'-end-labeled DNA probe containing the consensus binding site of interest. Samples were resolved by electrophoresis on a retardation $6 \%$ polyacrylamide gel (cat. no. \#EC6365BOX; Life Technologies, Thermo Fisher Scientific, Inc.) and transferred to a nylon membrane (cat. no. \#LC2003; Novex, Thermo Fisher Scientific, Inc.). The electrophoresis unit was filled with $0.5 \mathrm{X}$ Tris/borate EDTA
(TBE; cat. no. \#B52, Thermo Fisher Scientific, Inc.) immediately below the bottom of the wells.

Gels were pre-run at $100 \mathrm{~V}$. During the pre-run, control DNA and sample DNA binding reactions were performed, according to the protocol provided in the manual. Once the pre-run of the gel was finished, $20 \mu \mathrm{l}$ of sample containing loading buffer was loaded onto the gel. The gel was run until the Bromophenol blue dye had migrated three-quarters of the way down the length of the gel. The free biotin control-DNA duplex migrated immediately behind the Bromophenol blue. Subsequently, the binding reactions were transferred to nylon membranes at $380 \mathrm{~mA}(100 \mathrm{~V})$ for $30 \mathrm{~min}$. This step was followed by the crosslink transfer of DNA to the membrane using a transilluminator for 10-15 min. The ECL method was applied to detect biotin-labeled DNA. The membrane was subsequently placed into a film cassette and exposed to X-ray film for 2-5 min. The biotin end-labeled DNA probe was detected using streptavidin conjugated to HRP and a chemiluminescent substrate. These extracts were incubated with a biotin 3'-or 5'-end-labeled DNA probe with an IL-6 consensus sequence.

Duplex IL-6 oligonucleotide sense and antisense sequences. Oligonucleotide sequences were synthesized by Integrated DNA Technologies, Inc. (Coralville, IA, USA). The oligo1-IL-6 sense sequence was prepared with the following characteristics: M.W. 9.330.1; GC content, 40.0; extinction coefficient, 321,200 1/(mole.cm); and Mfg ID, M189229415. The oligol-IL-6 antisense strand was prepared with these characteristics: M.W. 8.547.6; GC content, 60.7; extinction coefficient, 248,800 1/(mole.cm); and Mfg ID, M189229416. The oligol-IL-6 sense and antisense strands consisted of the following sequences, respectively: 5'-CTGAGAAAGGAG ACATGTAACAAGAGTAAC-3' and 3'-CGACTGCTC GACGTCCGTGTCTTGGTCA-5'. The control biotin duplex had the following characteristics: 100 nmol, M.W. 18,664.5; sequences: 5'-/5Biosg/CTGAGAAAGGAGACATGT AACAAGAGTAAC-3' (sense) and 5'-/5Biosg/ACT GGTTCTGTGCCTGCAGCTCGTCAG C-3' (antisense), and those for the control non-biotin complex were: $100 \mathrm{nmol}$, M.W. 17,877.7; sequences: 5'-CTGAGAAAGGAGACATGTAAC AAGAGTAAC-3' (sense) and 5'-ACTGGTTCTGTGCCT GCAGCTCGTCAGC-3' (antisense). The company Active Motif also provided unlabeled control DNA target (non-biotin) for use in competition experiments to verify the specificity of the DNA-protein complex. The control nuclear extract was also included in the kit.

Histone H3K9 demethylase activity assay. The quantification of H3K9-specific histone demethylase activity was performed using the EpiSeeker Histone H3 (K9) Demethylase Activity Quantification Assay kit (cat. no.\#113458; Abcam), according to the manufacturer's protocol.

Statistical analysis. All experiments were performed in triplicate, and $\mathrm{P}<0.05$ was considered to indicate a statistically significant value. Data analysis was performed using a one-way analysis of variance (ANOVA) unpaired $t$-test (GraphPad Prism; GraphPad Software, San Diego, CA, USA). 


\section{Results}

A panel of 12 human inflammatory cytokines from the Human Inflammatory Cytokines Multi-Analyte ELISArray kit (Qiagen) were tested. Even though the goal of the present study was to make a comparison of cytokine expression primarily between the JJ012 human chondrosarcoma cell line and $\mathrm{C} 28$ chondrocytes, the results for the adult mesenchymal cell line were also included in the text, as well as those of the co-culture experiments. The experimental data pointed towards an absence of any differences in the expression of the cytokines among the cell lines, with the exception of IL-6. The expression of IL-6 in the JJ012 human chondrosarcoma cells was 86-fold lower compared with the C28 chondrocytes, based on the ELISA results. A 53-fold downregulation of IL-6 expression in co-culture of chondrosarcoma cells and C28 chondrocytes was demonstrated compared with the $\mathrm{C} 28$ chondrocytes (Fig. 1). The expression of IL-6 was 56-fold lower compared with mesenchymal adult cells, and a 65-fold downregulation of IL-6 expression in co-culture of mesenchymal stem cells and JJ012 cells was observed in comparison with its expression in mesenchymal cells. PRP-1 did not affect the levels of IL-6 (data not shown). From these experiments, it is possible to conclude that IL-6 manifested itself as an anti-inflammatory and, possibly, as an anti-tumorigenic factor.

To explore protein-DNA interactions leading to such differences, a gel-shift chemiluminescent assay was performed (Fig. 2). As shown in Fig. 2, the commercially supplied control is shown in lanes 1-3: In lane 2, the band-shift was observed due to the presence of biotinylated control and control nuclear extract. In lane 1, in the absence of the control nuclear extract, no shift was observed, since the reaction lacked protein to bind to the oligo-IL-6 DNA, and thereby to cause a shift. In lane 2, the target protein was present in the control nuclear extract, and bound to the biotin-labeled control DNA, thereby causing a shift compared with the reaction in lane 1 . In lane 3 , no shift was detected: The excess of unlabeled control DNA competed for binding of the target protein in the control nuclear extract. This experiment verified that the signal observed in lane 2 resulted from specific DNA-protein interactions.

Gel-shifts were also observed for chondrosarcoma cells and chondrocytes in the lanes with nuclear cell extract and oligo-IL-6 DNA. Notably, the DNA-protein complex in C28 cells was revealed to be markedly larger compared with chondrosarcoma cells: In the experimental samples, shown in lanes 4-6 for the JJ012 chondrosarcoma cells and lanes 7-9 for the C28 chondrocytes, band-shifts were observed in lanes 5 and 8 for the JJ012 and the C28 cells, respectively. Those lanes featuring gel-shifts included oligo-IL-6-biotinylated duplex with corresponding extracts either from the JJ012 or C28 cells, and the protein present in those nuclear extracts was able to bind to the IL-6 DNA. Lanes 4 and 7 featured oligo-IL-6-biotinylated duplex; lanes 6 and 9 featured oligo-IL- 6 biotinylated with corresponding nuclear extract and unlabeled IL-6 control, respectively, and therefore no shifts were observed in those lanes. Most importantly, in lane 8 for the C28 chondrocytes, the shifted band pertaining to the DNA-protein complex was much larger compared with that in lane 5 for the human JJ012 chondrosarcoma cells.

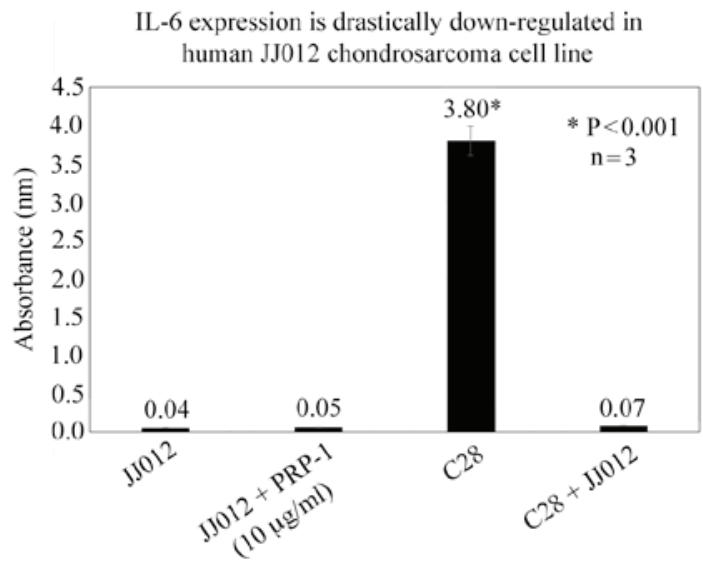

Figure 1. Comparison between the expression levels of IL-6 in chondrosarcoma sarcoma adult and the C28 chondrocyte cell line. IL- 6 expression was markedly downregulated in the human JJ012 chondrosarcoma cell line compared with the $\mathrm{C} 28$ chondrocytes.

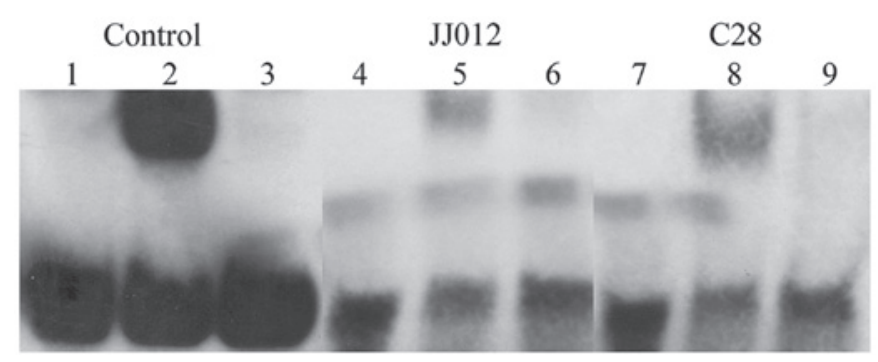

Figure 2. Electrophoretic mobility shift assay reveals differences between IL-6 DNA-protein complexes in human JJ012 chondrosarcoma and C28 chondrocyte nuclear extracts. The protein IL-6-DNA complex was markedly larger in C28 chondrocytes compared with the JJ012 cell line. The lanes were loaded as follows: Lane 1, biotinylated control; lane 2, biotinylated control + control nuclear extract; lane 3 , biotinylated control + control nuclear extract + unlabeled control DNA; lane 4, oligo-IL-6 duplex biotinylated; lane 5, oligo-IL-6 duplex biotinylated + JJ012 extract; lane 6, oligo-IL-6 duplex biotinylated + JJ012 + unlabeled oligo IL-6 control; lane 7, oligo-IL-6 duplex biotinylated; lane 8 , oligo-IL-6 duplex biotinylated + C28 extract; lane 9, oligo-IL-6 biotinylated DNA + C28 extract + unlabeled oligo-IL-6 control. IL-6, interleukin-6.

Western blot analysis demonstrated upregulated expression levels of SOCS3 in chondrocytes compared with the JJ012 chondrosarcoma cells (Fig. 3). Addition of PRP-1 restored the expression of SOCS3 in the JJ102 cells in a dose-responsive manner. No marked differences in the expression levels of signal transducer and activator of transcription 3 (STAT3) or Smad2 proteins were observed between the JJ012 cells and the C28 chondrocytes. SOCS3, in certain tumors, has been revealed to be a tumor suppressor, for example, in breast cancer cells, and its expression was determined to be independent of $\operatorname{STAT}(28,29)$. In the present study, a marked upregulation in the level of TGF- $\beta$ was observed in chondrocytes, contrasted with a marked downregulation of TGF- $\beta$ expression in the chondrosarcoma cells (Fig. 3). Addition of PRP-1 failed to restore the expression levels of TGF- $\beta$.

The expression levels of regulatory proteins of the Hedgehog and Hippo signaling pathways in the human JJ012 chondrosarcoma cell line are shown in Figs. 4 and 5, respectively. PRP-1 


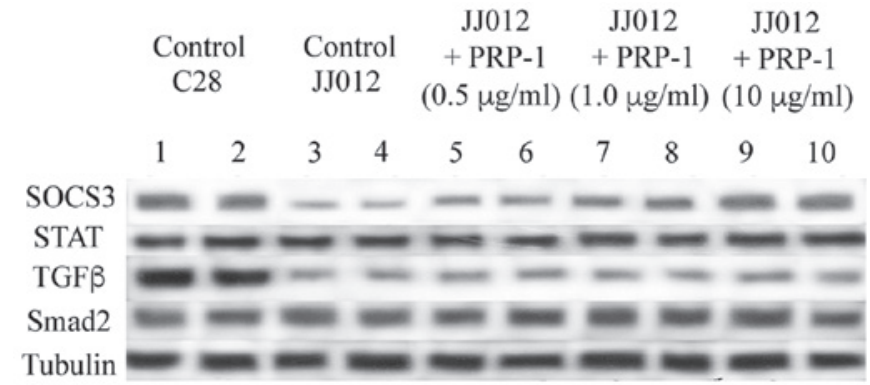

Figure 3. PRP-1 upregulates SOCS3 expression in a dose-responsive manner in the human chondrosarcoma JJ012 cell line. The tumor suppressor, SOC3, was markedly downregulated in the human JJ012 cell line compared with C28 chondrocytes. Addition of PRP-1 restored the expression of SOC3 (the band was detected at $\sim 30 \mathrm{kDa}$ ). The expression of TGF- $\beta$ (band detected at $25 \mathrm{kDa}$ ) was reduced in the $\mathrm{C} 28$ cells, and although it was markedly higher in $\mathrm{C} 28$ chondrocytes, PRP-1 failed to elicit any effects on TGF- $\beta$ expression. No differences in STAT or Smad2 protein expression were identified between the $\mathrm{C} 28$ and JJ012 cell lines. All primary antibodies were used at 1:1,000 dilution, and secondary antibodies at 1:5,000. $\alpha$-tubulin was used as a protein loading control, and each pairing of lanes (1-10) represents the identical experiment performed in duplicate. SOCS3, suppressor of cytokine signaling 3; STAT, signal transducer and activator of transcription 3; PRP-1, proline-rich polypeptide 1 ; TGF- $\beta$, transforming growth factor- $\beta$.

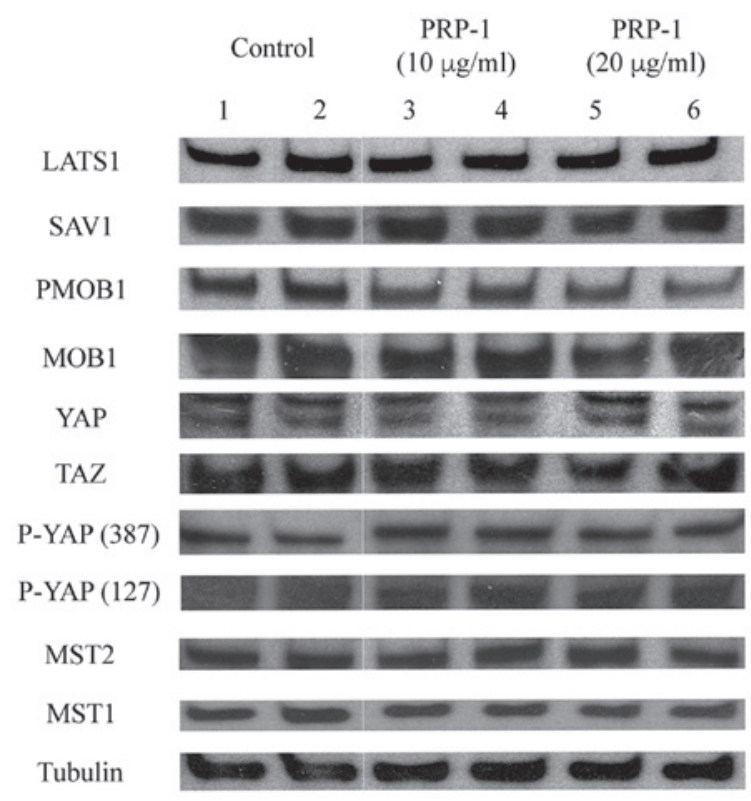

Figure 4. The expression of oncogene and tumor-suppressor proteins of the Hippo signaling pathway in the human JJ012 chondrosarcoma cell line. PRP-1 did not exert any effect on the components of the Hippo signaling pathway, except for a modest upregulation in the levels of phosphorylated Yap (387). $\alpha$-tubulin was used as a protein loading control, and each pairing of lanes (1-10) represents the identical experiment performed in duplicate. PRP-1, proline-rich polypeptide 1; MST1/2, Ste 20 family protein kinases.

did not exert any effect on the tumor suppressors or oncoproteins of those respective pathways. Other western blot analyses were performed to measure the expression levels of the proteins TET1 and TET2 in the human JJ012 chondrosarcoma cell line, since their downregulation is an early event in cell transformation, and these proteins act predominantly as tumor suppressors. A dose-responsive upregulation in the expression levels of the TET1 and TET2 proteins was observed (Fig. 6). From the epigenetic standpoint, PRP-1 was revealed to inhibit H3K9

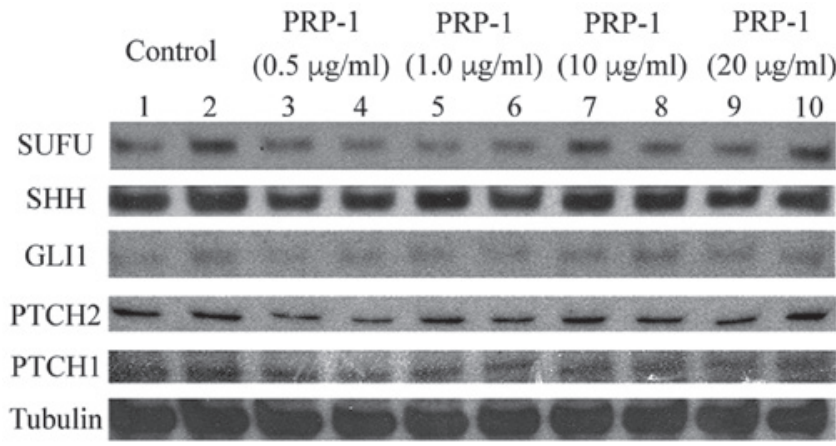

Figure 5. The expression of oncogene and tumor-suppressor proteins of the Hedgehog signaling pathway in the human JJ012 chondrosarcoma cell line. As for the Hippo pathway, PRP-1 did not exert any marked effect on the components of the Hedgehog signaling pathway. $\alpha$-tubulin was used as a protein loading control, and each pairing of lanes (1-10) represents the identical experiment performed in duplicate. PRP-1, proline-rich polypeptide 1; SUFU, suppressor of fused homolog; SHH, Sonic Hedgehog; PTCH1/2, Patch homology $1 / 2$.

\begin{tabular}{|c|c|c|c|c|c|c|c|c|}
\hline & \multicolumn{2}{|c|}{ Control } & \multicolumn{2}{|c|}{$\begin{array}{c}\text { PRP-1 } \\
(1 \mu \mathrm{g} / \mathrm{ml})\end{array}$} & \multicolumn{2}{|c|}{$\begin{array}{c}\text { PRP-1 } \\
(10 \mu \mathrm{g} / \mathrm{ml})\end{array}$} & \multicolumn{2}{|c|}{$\begin{array}{c}\text { PRP-1 } \\
(20 \mu \mathrm{g} / \mathrm{ml})\end{array}$} \\
\hline & 1 & 2 & 3 & 4 & 5 & 6 & 7 & 8 \\
\hline TET1 & & & & & & & & \\
\hline TET2 & & & & & & & & \\
\hline abulin & & & & & & & & \\
\hline
\end{tabular}

Figure 6. Upregulation of the expression levels of the TET1/TET2 proteins by PRP-1 in the human chondrosarcoma JJ012 cell line. PRP-1 upregulated the expression of the tumor suppressors TET1 and TET1 in a dose-responsive manner. Bands were detected at $224 \mathrm{kDa}$. $\alpha$-tubulin was used as a protein loading control, and each pairing of lanes (1-10) represents the identical experiment performed in duplicate. PRP-1, proline-rich polypeptide 1; TET $1 / 2$, ten-eleven-translocation methylcytosine dioxygenase $1 / 2$.

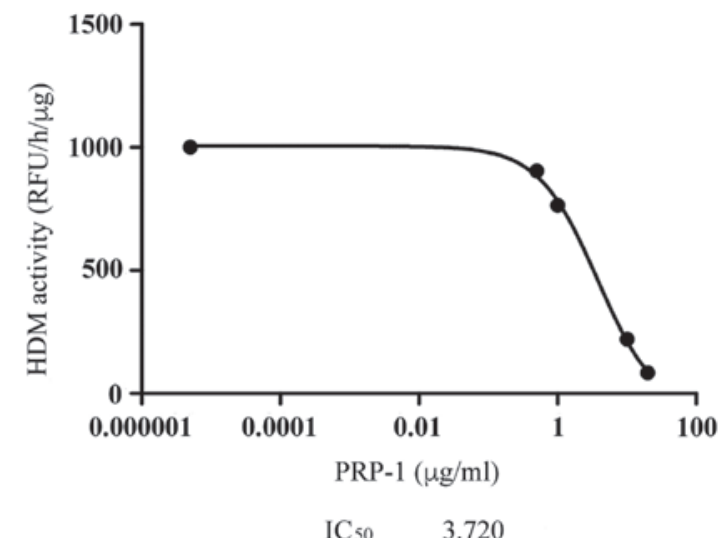

Figure 7. Inhibition of H3K9 demethylase activity mediated by PRP-1 in the human JJ012 chondrosarcoma cell line. H3K9 methylation is eliminated from the promoters of the inflammation-associated genes. PRP-1 inhibited H3K9 demethylase activity with an $\mathrm{IC}_{50}$ (concentration required to give malf-maximal inhibition) value of $3.72 \mu \mathrm{g} / \mathrm{ml}$ in the human JJ012 chondrosarcoma cell line. PRP-1, proline-rich polypeptide 1; HDM, histone demethylase; RFU, relative fluorescence units.

demethylase activity with an $\mathrm{IC}_{50}$ (concentration required to give half-maximal inhibition) value of $3.72 \mu \mathrm{g} / \mathrm{ml}$ in the human JJ012 chondrosarcoma cell line (Fig. 7). 


\section{Discussion}

The marked downregulation of IL-6 expression in the human JJ012 chondrosarcoma cell line compared with C28 chondrocytes prompted further elucidation of the putative mechanism involved in this process. Reduced expression of IL-6 as a growth inhibitor and differentiation factor has been reported in certain tumors, and this has been linked with the compromised differentiation program for a number of carcinoma and leukemia cell lines (30-33). Tumor rejection and long-term survival rates have been reported in response to IL-6. It is important to mention that IL-6 manifests itself either as an anti-inflammatory or a pro-inflammatory cytokine (30-33). IL-6 expression has often been determined to be within normal levels in well-differentiated forms of thyroid carcinoma, although it was markedly suppressed in undifferentiated forms of thyroid carcinomas (34). A sarcomatoid component is present in these types of tumors. Anaplastic thyroid carcinomas (ATCs) of sarcomatoid appearance are characterized by spindle cells and giant cells, which are the most frequent cells observed in ATC. Primary sarcomas simulating a sarcomatoid ATC have been reported in case reports for many types of sarcoma, including chondrosarcoma (35). The gel-shift assay revealed the presence of a larger protein IL-6-DNA complex in the C28 chondrocytes compared with the chondrosarcoma cells. The characteristics and origin of the proteins, and the mechanisms which are responsible for such differences, have yet to be investigated. Another aspect that deserves further investigation is the regulation of IL- 6 by miRNAs, and their involvement in the regulation of the differentiation program. Our group has previously demonstrated that the downregulation of miR $181 \mathrm{~b}$ leads to the downregulation of its target protein, IL-6 (36).

The upregulation of SOCS3, TET1 and TET2 expression in a dose-dependent manner by PRP-1 further demonstrates the ability of this cytokine peptide to upregulate tumor suppressor genes in general (8-10). Even though PRP-1 did not exert any effect on tumor suppressors or oncoproteins of the Hippo or Hedgehog signaling pathways, there was an important conclusion to consider: The inhibition of tumor suppressors by PRP-1 depends on the pathway these tumor suppressors represent or are involved in. TET1 and TET2 act predominantly as tumor suppressors. Re-introduction and overexpression of these proteins is able to restore the $5 \mathrm{hmC}$ content, and to suppress invasion and growth in certain tumors (37-41). A previous report revealed that a deficiency in SOCS3 may promote tumor development, since it has tumor suppressor functions in numerous cancer cell types (29). It was demonstrated that SOCS3, in certain circumstances, acts as a regulator of pathways and processes that are unrelated to the STAT signaling cellular pathways. SOCS3 attenuates pro-inflammatory signaling (42), and its deficiency promotes inflammation (43). However, the role exerted by SOCS3 during bone inflammation is complex, and effects may work in opposition to each other; therefore, knowledge of further mechanistic details concerning the SOCS3 pathway are necessary for a better understanding of the processes of various bone inflammatory diseases. The epigenetic regulation of inflammation linked with oncogenesis has a central role. It is known that $\mathrm{H} 3 \mathrm{~K} 9$ methylation is eliminated from the promoters of inflammation-associated genes (44), and, therefore the mechanisms underpinning the inhibition of $\mathrm{H} 3 \mathrm{~K} 9$ demethylases merit further attention. The ability of PRP-1 to inhibit H3K9 demethylase activity was previously reported by our group (8), and a possible mechanism of the peptide's function in the upregulation of tumor suppressors and in the downregulation of oncoproteins was proposed. In the present study, the PRP-1-mediated inhibition of H3K9 demethylase was associated with the process of inflammation, and the $\mathrm{IC}_{50}$ value has been demonstrated.

In summary, cytokines produced in the tumour microenvironment have an important role in cancer pathogenesis and in the inhibition of disease progression. IL-6 family cytokines appear to be double-edged swords, since the development of bone cancers may be either prevented or enhanced by this family of cytokines, depending on the cell type, the stage of the tumor and the bone environment (45). Observed downregulation of IL-6 in the human JJ012 chondrosarcoma cell line compared with the chondrocytes supports the hypothesis that IL-6 is acting as a differentiation/anti-inflammatory factor in this cellular context, indicating the possibility that a factor(s) or interacting proteins in the tumor have the ability to inhibit IL-6 expression. Understanding the differences in their expression patterns between the normal and malignant states, and the signaling pathways that lead to these differences, require further investigation as important targets for future therapeutic interventions. Metastatic chondrosarcoma does not respond to conventional therapies and the search for new therapeutic approaches is urgent $(2-10,36,46)$. The ability of PRP-1 as an antiproliferative agent to restore the expression of anti-inflammatory cytokines with tumor suppressor function proves the importance of this neuropeptide for future clinical consideration.

\section{Acknowledgements}

The present study was supported in part by the Ratcliffe Foundation gift to Miami Center of Orthopedic Research and Education (Miami CORE).

\section{References}

1. Landskron G, De la Fuente M, Thuwajit P, Thuwajit C and Hermoso MA: Chronic inflammation and cytokines in the tumor microenvironment. J Immunol Res 2014: 149185, 2014.

2. Mirra J: Bone Tumors: Clinical, radiologic, and pathologic correlations. Lea and Febiger, Philadelphia, PA, 1989.

3. Galoian K, Scully S, McNamara G, Flynn P and Galoyan A Antitumorigenic effect of brain proline rich polypeptide-1 in human chondrosarcoma. Neurochem Res 34: 2117-2121, 2009.

4. Galoian K, Scully S and Galoyan A: Myc-oncogene inactivating effect by proline rich polypeptide (PRP-1) in chondrosarcoma JJ012 cells. Neurochem Res 34: 379-385, 2009.

5. Galoian K, Temple HT and Galoyan A: Cytostatic effect of the hypothalamic cytokine PRP-1 is mediated by mTOR and cMyc inhibition in high grade chondrosarcoma. Neurochem Res 36: 812-818, 2011.

6. Galoian KA, Temple HT and Galoyan A: Cytostatic effect of novel mTOR inhibitor, PRP-1 (galarmin) in MDA231 (ER-) breast carcinoma cell line. PRP-1 inhibits mesenchymal tumors. Tumour Biol 32: 745-51, 2011.

7. Galoian K, Temple HT and Galoyan A: mTORC1 inhibition and ECM-cell adhesion-independent drug resistance via P13K-AKT and PI3K-RAS-MAPK feedback loops. Tumour Biol 33: 885-90, 2012.

8. Galoian K, Qureshi A, D'ippolito G, Schiller PC, Molinari M, Johnstone AL, Brothers SP, Paz AC and Temple HT: Epigenetic regulation of embryonic stem cell marker miR302C in human chondrosarcoma as determinant of antiproliferative activity of proline-rich polypeptide 1. Int J Oncol 47: 465-472, 2015. 
9. Galoian K, Qureshi A, Wideroff G and Temple HT: Restoration of desmosomal junction protein expression and inhibition of H3K9-specific histone demethylase activity by cytostatic proline-rich polypeptide-1 leads to suppression of tumorigenic potential in human chondrosarcoma cells. Mol Clin Oncol 3 : 171-178, 2015.

10. Galoian K, Guettouche T, Issac B, Qureshi A and Temple HT: Regulation of onco and tumor suppressor miRNAs by mTORC 1 inhibitor PRP-1 in human chondrosarcoma. Tumour Biol 35: 2335-2341, 2014.

11. Yang H, Liu Y, Bai F, Zhang JY, Ma SH, Liu J, Xu ZD, Zhu HG, Ling ZQ, Ye D, et al: Tumor development is associated with decrease of TET gene expression and 5-methylcytosine hydroxylation. Oncogene 32: 663-669, 2013.

12. He YF, Li BZ, Li Z, Liu P, Wang Y, Tang Q, Ding J, Jia Y, Chen Z, Li L, et al: Tet-mediated formation of 5-carboxylcytosine and its excision by TDG in mammalian DNA. Science 333: 1303-1307, 2011.

13. Ito S, Shen L, Dai Q, Wu SC, Collins LB, Swenberg JA, He C and Zhang Y: Tet proteins can convert 5-methylcytosine to 5-formylcytosine and 5-carboxylcytosine. Science 333: 1300-1303, 2011.

14. Chmielecki J and Meyerson M: DNA sequencing of cancer: What have we learned? Annu Rev Med 65: 63-79, 2014

15. Xu W, Yang H, Liu Y, Yang Y, Wang P, Kim SH, Ito S, Yang C, Wang P, Xiao MT, et al: Oncometabolite 2-hydroxyglutarate is a competitive inhibitor of $\alpha$-ketoglutarate-dependent dioxygenases. Cancer Cell 19: 17-30, 2011.

16. Tahiliani M, Koh KP, Shen Y, Pastor WA, Bandukwala H, Brudno Y, Agarwal S, Iyer LM, Liu DR, Aravind L and Rao A: Conversion of 5-methylcytosine to 5-hydroxymethylcytosine in mammalian DNA by MLL partner TET1. Science 324: 930-935, 2009.

17. Suijker J, Oosting J, Koornneef A, Struys EA, Salomons GS, Schaap FG, Waaijer CJ, Wijers-Koster PM, Briaire-de Bruijn IH, Haazen L, et al: Inhibition of mutant IDH1 decreases D-2-HG levels without affecting tumorigenic properties of chondrosarcoma cell lines. Oncotarget 6: 12505-12519, 2015.

18. Haseeb A, Makki MS and Haqqi TM: Modulation of ten-eleven translocation 1 (TET1), Isocitrate Dehydrogenase (IDH) expression, $\alpha$-Ketoglutarate $(\alpha-K G)$, and DNA hydroxymethylation levels by interleukin-1 $\beta$ in primary human chondrocytes. J Biol Chem 289: 6877-6885, 2014.

19. Deel MD, Li JJ, Crose LE and Linardic CM: A review: Molecular aberrations within hippo signaling in bone and soft-tissue sarcomas. Front Oncol 5: 190,2015.

20. Tiet TD, Hopyan S, Nadesan P, Gokgoz N, Poon R, Lin AC, Yan T, Andrulis IL, Alman BA and Wunder JS: Constitutive hedgehog signaling in chondrosarcoma up-regulates tumor cell proliferation. Am J Pathol 168: 321-30, 2006.

21. Lu L, Li Y, Kim SM, Bossuyt W, Liu P, Qiu Q, Wang Y, Halder G, Finegold MJ, Lee JS and Johnson RL: Hippo signaling is a potent in vivo growth and tumor suppressor pathway in the mammalian liver. Proc Natl Acad Sci USA 107: 1437-1442, 2010.

22. Eisinger-Mathason TS, Mucaj V, Biju KM, Nakazawa MS Gohil M, Cash TP, Yoon SS, Skuli N, Park KM, Gerecht S and Simon MC: Deregulation of the Hippo pathway in soft-tissue sarcoma promotes FOXM1 expression and tumorigenesis. Proc Natl Acad Sci USA 112: E3402-E3411, 2015.

23. Mohamed AD, Tremblay AM, Murray GI and Wackerhage $H$ The Hippo signal transduction pathway in soft tissue sarcomas. Biochim Biophys Acta 1856: 121-129, 2015.

24. Li R, Cai L, Ding J, Hu CM, Wu TN and Hu XY: Inhibition of hedgehog signal pathway by cyclopamine attenuates inflammation and articular cartilage damage in rats with adjuvant-induced arthritis. J Pharm Pharmacol 67: 963-971, 2015.

25. Nakashima H, Nakamura M, Yamaguchi H, Yamanaka $N$, Akiyoshi T, Koga K, Yamaguchi K, Tsuneyoshi M, Tanaka M and Katano M: Nuclear factor-kappaB contributes to hedgehog signaling pathway activation through sonic hedgehog induction in pancreatic cancer. Cancer Res 66: 7041-7049, 2006

26. Zacharias WJ, Li X, Madison BB, Kretovich K, Kao JY, Merchant JL and Gumucio DL: Hedgehog is an anti-inflammatory epithelial signal for the intestinal lamina propria. Gastroenterology 138: 2368-2377, 2377.e1-e4, 2010.
27. Taniguchi K, Wu LW, Grivennikov SI, de Jong PR, Lian I, Yu FX, Wang K, Ho SB, Boland BS, Chang JT, et al: A gp130-Src-YAP module links inflammation to epithelial regeneration. Nature 519: 57-62, 2015.

28. Duh QY and Grossman RF: Thyroid growth factors, signal transduction pathways, and oncogenes. Surg Clin North Am 75: 421-437, 1995.

29. Barclay JL, Anderson ST, Waters MJ and Curlewis JD: SOCS3 as a tumor suppressor in breast cancer cells, and its regulation by PRL. Int J Cancer 124: 1756-1766, 2009.

30. Revel M: Growth regulatory functions of IL6 and antitumor effects. Res Immunol 143: 769-773, 1992.

31. Givon T, Slavin S, Haran-Ghera N, Michalevicz R and Revel M Antitumor effects of human recombinant interleukin- 6 on acute myeloid leukemia in mice and in cell cultures. Blood 79 2392-2398, 1992

32. Basolo F, Fiore L, Pollina L, Fontanini G, Conaldi PG and Toniolo A: Reduced expression of interleukin 6 in undifferentiated thyroid carcinoma: in vitro and in vivo studies. Clin Cancer Res 4: 381-387, 1998.

33. Mulé JJ, McIntosh JK, Jablons DM and Rosenberg SA: Antitumor activity of recombinant interleukin 6 in mice. J Exp Med 171: 629-636, 1990.

34. Ragazzi M, Ciarrocchi A, Sancisi V, Gandolfi G, Bisagni A and Piana S: Update on anaplastic thyroid carcinoma: Morphological, molecular, and genetic features of the most aggressive thyroid cancer. Int J Endocrinol 2014: 790834, 2014.

35. Tseleni-Balafouta S, Arvanitis D, Kakaviatos $\mathrm{N}$ and Paraskevakou H: Primary myxoid chondrosarcoma of the thyroid gland. Arch Pathol Lab Med 112: 94-96, 1988.

36. Galoian K, Guettouche T, Issac B, Navarro L and Temple HT: Lost miRNA surveillance of Notch, IGFR pathway-road to sarcomagenesis. Tumour Biol 35: 483-492, 2014

37. Sarkar S, Horn G, Moulton K, Oza A, Byler S, Kokolus S and Longacre M: Cancer development, progression, and therapy: An epigenetic overview. Int J Mol Sci 14: 21087-21113, 2013.

38. Lian CG, Xu Y, Ceol C, Wu F, Larson A, Dresser K, Xu W, Tan L, Hu Y, Zhan Q, et al: Loss of 5-hydroxymethylcytosine is an epigenetic hallmark of melanoma. Cell 150: 1135-1146, 2012.

39. Fan M, He X and Xu X: Restored expression levels of TET1 decrease the proliferation and migration of renal carcinoma cells. Mol Med Rep 12: 4837-4842, 2015.

40. Cimmino L, Dawlaty MM, Ndiaye-Lobry D, Yap YS, Bakogianni S, Yu Y, Bhattacharyya S, Shaknovich R, Geng H, Lobry C, et al: TET1 is a tumor suppressor of hematopoietic malignancy. Nat Immunol 16: 889, 2015.

41. Zhang Q, Zhao K, Shen Q, Han Y, Gu Y, Li X, Zhao D, Liu Y, Wang C,Zhang X, et al: Tet2 is required to resolve inflammation by recruiting Hdac2 to specifically repress IL-6. Nature 525 : 389-393, 2015.

42. Jo D, Liu D, Yao S, Collins RD and Hawiger J: Intracellular protein therapy with SOCS3 inhibits inflammation and apoptosis. Nat Med 11: 892-898, 2005

43. Qin H, Holdbrooks AT, Liu Y, Reynolds SL, Yanagisawa LL and Benveniste EN: SOCS3 deficiency promotes M1 macrophage polarization and inflammation. J Immunol 189: 3439-3448, 2012.

44. Villeneuve LM, Reddy MA, Lanting LL, Wang M, Meng L and Natarajan R: Epigenetic histone H3 lysine 9 methylation in metabolic memory and inflammatory phenotype of vascular smooth muscle cells in diabetes. Proc Natl Acad Sci USA 105 9047-9052, 2008.

45. Blanchard F, Duplomb L, Baud'huin M and Brounais B: The dual role of IL-6-type cytokines on bone remodeling and bone tumors. Cytokine Growth Factor Rev 20: 19-28, 2009.

46. Clark JC, Akiyama T, Dass CR and Choong PF: New clinically relevant, orthotopic mouse models of human chondrosarcoma with spontaneous metastasis. Cancer Cell Int 10: 20, 2010. 